\title{
Consecuencias del amonio en la fatiga central en atletas, posible efecto neuroprotector del ejercicio
}

\section{Ammonium consequences in athletes' central fatigue and its possible neuroprotection effect thanks to physical activity \\ Consequências do amônio na fadiga central em atletas, possível efeito neuroprotetor do exercício}

\author{
Javier Porras-Álvarez, L. Educación Física, MSc. PhD (c)*
}

\section{Resumen}

Introducción. La fatiga central en el deporte está asociada a los efectos del amonio. La principal fuente de producción de amonio durante el ejercicio es el músculo esquelético. El amonio se genera como consecuencia del metabolismo energético, debido a la oxidación de aminoácidos y a la desaminación del nucleótido de adenosin trifosfato. Objetivo. Presentar una reflexión sobre el efecto del amonio durante el ejercicio de alta intensidad y su relación con la fatiga central en atletas. Discusión. Durante el ejercicio, la concentración de amonio alcanza valores superiores a $200 \mu \mathrm{M}$ (micromolar); sin embargo, en un adulto promedio se considera que valores superiores a $60 \mu \mathrm{M}$ en sangre manifiestan un trastorno por hiperamonemia. El amonio influye en la disminución del rendimiento en atletas y está asociado con los efectos nocivos para la salud en pacientes con encefalopatía hepática. Conclusiones. La práctica del ejercicio físico genera neuroprotección contra las altas concentraciones de amonio en el cerebro, pues, durante el ejercicio con altas concentraciones de amonio, los atletas no presentan los síntomas de pacientes con encefalopatía hepática, lo que implica adaptaciones metabólicas que juegan un papel importante en el metabolismo del amonio en el cerebro. [Porras-Álvarez J. Consecuencias del amonio en la fatiga central en atletas, posible efecto neuroprotector del ejercicio. MedUNAB. 2018;21(1): 115-121 doi: $10.29375 / 01237047.3394]$.

Palabras clave: Fatiga; Compuestos de Amonio

\footnotetext{
* Licenciado en ciencias de la Educación Física, magíster en Ciencias de la Actividad Física y Deporte, doctorando en Medicina del Deporte, doctorando en Ciencias Biomédicas. Docente de la Universidad Pedagógica y Tecnológica de Colombia, grupo de investigación FIMED, Tunja, Boyacá, Colombia.
}

Correspondencia: Javier Porras-Álvarez. Vereda Molino, municipio de Saboyá. Celular: +57 3143029381. E-mail: porrasalvarez@gmail.com; javier. porras@uptc.edu.co. 
Cuaternario; Entrenamiento de Resistencia; Ejercicio; Sistema Inmunológico; Ácido Úrico; Encefalopatía Hepática.

\section{Abstract}

Introduction. Central fatigue in sports training is associated with ammonium effects within the human body. The ammonium main production source during physical training is located in skeletal muscles and it is generated as a result of energy metabolism. This process is caused by amino acids oxidation and adenosine triphosphate nucleotide deamination. Objective. This article's objective is to present an analysis regarding ammonium effects when high intensity sports are performed and its relation with central fatigue in athletes. Discussion. When high intensity sport practices are performed, ammonium concentration levels can reach values higher than $200 \mu \mathrm{M}$ (micromolar). However, it is considered that an average adult with ammonium levels higher than $60 \mu \mathrm{M}$ evidences a hyperammonemia disorder. Ammonium has direct influence in the decline of athletic performance and it is associated with harmful effects for hepatic encephalopathy patients. Conclusions. Physical activity practice creates neuroprotection against highquantities of ammonium in the brain. Although in physical practices athletes have high amounts of ammonium, they do not show symptoms related to hepatic encephalopathy; thus, this situation implies that metabolic adaptations have an important role within ammonium metabolism in the brain. [Porras-Alvarez J. Ammonium consequences in athletes' central fatigue and its possible neuroprotection effect thanks to physical activity. MedUNAB. 2018;21(1):115-121 doi: 10.29375/01237047.3394].

Key words: Fatigue; Quaternary Ammonium Compounds; Resistance Training; Exercise; Immune System; Uric Acid; Hepatic Encephalopathy.

\section{Resumo}

Introdução. A fadiga central no esporte está associada aos efeitos do amônio. A fonte de produção do amônio mais importante durante o exercício é o músculo esquelético. O amônio é gerado como consequência do metabolismo energético, devido à oxidação dos aminoácidos e à desaminação do trifosfato de adenosina. Objetivo. Apresentar uma reflexão sobre o efeito do amônio durante o exercício de alta intensidade e sua relação com a fadiga central em atletas. Discussão. Durante o exercício, a concentração do amônio atinge valores superiores a $200 \mu \mathrm{M}$ (micromol); no entanto, em um adulto médio, considera-se que valores superiores a $60 \mu \mathrm{M}$ no sangue mostram um distúrbio por hiperamonemia. $\mathrm{O}$ amônio influencia a diminuição do desempenho em atletas e está associado a efeitos nocivos para a saúde em pacientes com encefalopatia hepática. Conclusões. A prática de exercício físico gera neuroproteção contra altas concentrações do amônio no cérebro, pois, durante o exercício com altas concentrações do amônio, os atletas não apresentam sintomas de encefalopatia hepática, o que implica adaptações metabólicas que desempenham um papel importante no metabolismo do amônio no cérebro. [PorrasÁlvarez J. Consequências do amônio na fadiga central em atletas, possível efeito neuroprotetor do exercício. MedUNAB. 2018;21(1):115-121 doi: 10.29375/01237047.3394].

Palavras chave: Fadiga; Compostos de Amônio Quaternário; Treinamento de Resistência; Exercício; Sistema Imunitário; Ácido Úrico; Encefalopatia Hepática.

\section{Introducción}

El entrenamiento diario de alta intensidad al que se someten los atletas induce cambios y adaptaciones en los diferentes órganos y sistemas del cuerpo humano, llevando al límite los parámetros bioquímicos y fisiológicos. No obstante, hay barreras que impiden, por ejemplo, ir más rápido, saltar más alto o realizar el gesto de forma perfecta. Aunque las marcas y el nivel técnico de los atletas mejoran continuamente, hay procesos bioquímicos que actúan como límites o barreras que impiden al atleta realizar marcas supra humanas. De esta forma, la fatiga se define como una disminución reversible de la capacidad de rendimiento. A esta se han atribuido causas centrales y periféricas, que generan en el atleta la incapacidad de seguir manteniendo una velocidad dada o mejorar el rendimiento físico.

Las causas periféricas hacen referencia a aquellas que ocurren, por ejemplo, a nivel muscular, relacionadas con: el porcentaje ideal del tipo de fibras musculares que requiere la modalidad deportiva; un adecuado nivel enzimático; una disminución de sustratos energéticos disponibles, como glucosa, ácidos grasos e incapacidad para metabolizar oxígeno; o a altas concentraciones de adenosín monofostato $(1,2)$. Por su parte, las causas centrales se refieren a las que ocurren en el cerebro. Sustancias o moléculas que se producen en el músculo, como producto del metabolismo energético, pueden causar fatiga central, convirtiéndose en una barrera o límite en el desempeño del atleta.

La fatiga central en los atletas se da por múltiples factores, bien sean fisiológicos, psicológicos, ambientales, o mecanismos neurohumorales y del 
metabolismo cerebral. Al respecto, en 2004, Nybo indica que la fatiga central es el último factor que establece el punto de agotamiento, debido a cambios metabólicos, circulatorios, de neurotransmisores, termodinámicos, o a otros trastornos de la homeostasis cerebral, asociados a la acumulación o al agotamiento de diferentes sustratos en el cerebro. Estos implican la serotonina, la dopamina, la noradrenalina, las interleucinas, la hipoglicemia, la hipertermia y también al amoniaco o amonio (1).

El amoniaco $\left(\mathrm{NH}_{3}\right)$ es un compuesto químico cuya molécula está formada por un átomo de nitrógeno y tres átomos de hidrógeno. En disolución acuosa, como el medio metabólico humano, este se transforma en ion amonio $\left(\mathrm{NH}_{4}^{+}\right)$. El amonio es, por naturaleza, tóxico, pues puede atravesar la barrera hematoencefálica, ingresar al sistema nervioso central (SNC) e impedir que las motoneuronas funcionen de manera adecuada, ocasionando fatiga central $(1,3)$.

Durante el ejercicio, los atletas producen grandes concentraciones de amonio, producto del metabolismo energético, compuesto que puede influir en la disminución del rendimiento atlético. Asimismo, altas concentraciones de amonio están asociadas a efectos nocivos para la salud, como se evidencia en los pacientes con encefalopatía hepática (4-6). Por tanto, el objetivo de este artículo es reflexionar acerca del efecto del amonio durante el ejercicio de alta intensidad y su relación con la fatiga central en atletas.

\section{Concentración y producción de amonio durante el ejercicio}

En seres humanos sanos, la concentración normal de amonio en sangre es de alrededor de $20 \mu \mathrm{M}$ (micromolar) hasta $60 \mu \mathrm{M}(1 \mu \mathrm{M}$ equivale a $1 \mu \mathrm{mol} / 1$ : micromole/litro). Por su parte, los atletas alcanzan concentraciones de amonio superiores a $200 \mu \mathrm{M}$ durante el ejercicio, tanto de alta intensidad como de larga duración, según lo reportado en varias investigaciones (4-6). Valores de amonio en sangre superior a $60 \mu \mathrm{M}$ son considerados un trastorno por hiperamonemia, asociada a pacientes con encefalopatía hepática (HE).

La principal fuente de producción de amonio $\left(\mathrm{NH}_{3}\right.$ y $\mathrm{NH}_{4}^{+}$), durante el ejercicio, se sitúa en el músculo esquelético. Este amonio se genera como consecuencia del metabolismo energético, debido, por una parte, a la oxidación de ciertos aminoácidos, principalmente alanina, aspartato, glutamina, isoleucina, leucina y valina $\mathrm{y}$, por otra parte, a la desaminación del nucleótido de adenosín trifosfato (ATP, del inglés adenosine triphosphate), nucleótido esencial en la producción de energía para la contracción muscular (2, 7-10). En los pacientes con HE, el amonio se genera, principalmente, en el intestino grueso, en la porción del colon, aunque también se genera amonio en otros órganos, como corazón, páncreas, estomago, pulmones, cerebro y riñón (11). En la Figura 1 se presentan las dos fuentes principales de producción de amonio en atletas y pacientes con HE.

El ejercicio de alta intensidad requiere, de forma inmediata, de grandes cantidades de energía. Para cubrir las necesidades energéticas de la contracción muscular, esta energía es proporcionada a través de la hidrolisis del ATP, un nucleótido esencial como fuente principal de energía química para la mayoría de las funciones celulares $(9,10)$. El ATP está formado por una base nitrogenada (adenina), un azúcar de tipo pentosa (ribosa), enlazados a tres grupos fosfato. También, es requerido para que se produzca el acortamiento de los filamentos de actina-miosina y

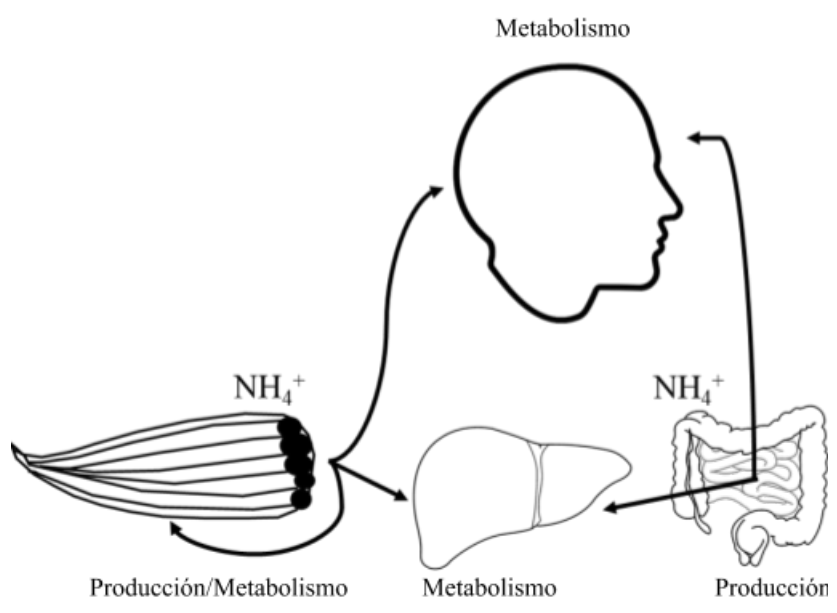

Figura 1. Producción de amonio durante el ejercicio en atletas y en pacientes con HE.

Durante el ejercicio de alta intensidad, la producción de amonio en los atletas se incrementa más de un $200 \%$ y se origina, principalmente, en el músculo esquelético en contracción. En los pacientes con HE, aproximadamente el $25 \%$ del amonio es generado en el sistema gastrointestinal. El amonio, generado tanto en músculo como intestinos, se metaboliza principalmente en el hígado, pero también en el músculo esquelético y en el cerebro.

Fuente: elaborado por autor 
generar la contracción muscular.

La energía se genera a partir del rompimiento de un grupo fosfato de la molécula de ATP, con la acción de la enzima ATPasa. El rompimiento de este enlace produce la energía para los procesos celulares, en este caso, para que se realice la contracción muscular. Consecuentemente, como producto de la reacción, se originan adenosín difosfato (ADP) y un grupo fosfato. El ADP es una molécula de adenosina con dos grupos fosfato, que se va acumulando a medida que se realiza el ejercicio, como producto de la reacción. La acumulación del ADP bloquea o inhibe la enzima ATPasa y, consecuentemente, disminuye la disponibilidad de energía para el músculo. Por tanto, se requiere obtener nuevamente un grupo fosfato, para reemplazar el fosfato liberado de la molécula de ATP. Este grupo fosfato se obtiene de reacciones metabólicas de otros compuestos, como fosfocreatina (PC), glucosa, ácidos grasos y determinados aminoácidos $(2,9-10,12)$.

Durante el ejercicio, las reservas de glucógeno y los compuestos ricos en energía, como la PC y la glucosa presentes en las células, se agotan rápidamente, a intensidades superiores al $80 \%$ del máximo consumo de oxígeno $\left(\mathrm{VO}_{2}\right)$. Como consecuencia, se activa la ruta de la enzima miokinasa (MK), la cual cataliza la reacción de dos ADP para formar un ATP. Como resultado de la reacción, queda adenosín monofosfato (AMP). Esto significa que las dos moléculas de ADP suman cuatro grupos fosfato, por lo que una molécula cede un grupo fosfato a la otra molécula. Esta es la reacción que cataliza la MK. Consecuentemente, se obtiene ATP y la otra molécula queda con un grupo fosfato (AMP). De esta manera, se dispone de una ruta metabólica alternativa para proporcionar energía de forma inmediata.

La consecuencia de la utilización de la ruta de la MK es la acumulación de AMP, un metabolito responsable de causar fatiga periférica, porque la acumulación de AMP bloquea o inhibe la acción de la enzima MK. Por esto, también se acumula ADP, un potente inhibidor de la ATPasa, generando así fatiga periférica $(9,10)$.

ElAMP es un desecho metabolito, del cual el organismo debe liberase a medida que se va produciendo (2, $9-10,13)$. No obstante, la eliminación del AMP implica el rompimiento de este nucleótido (AMP) o su desaminación, es decir, la eliminación del grupo $\alpha$-amino de los aminoácidos, en la cual se incluyen dos tipos de reacciones químicas: transaminación y aminación. Este proceso produce amonio, cuya acumulación, en consecuencia, aumenta durante el ejercicio de alta intensidad (Figura 2). Si se agotan las reservas de glucógeno y/o la disponibilidad de glucosa es baja, la concentración de amonio aumenta más rápidamente durante el ejercicio $(1,8,14-16)$.

\section{Producción de amonio en pacientes con encefalopatía hepática}

Se han evidenciado grandes concentraciones de amonio en pacientes con HE. Esta es una grave complicación de la cirrosis hepática, donde el daño ocasionado en el hígado afecta el metabolismo del amonio, causa una excesiva acumulación y alcanza valores superiores o iguales a $60 \mu \mathrm{M}$. Este fenómeno es considerado hiperamonemia, produce efectos perjudiciales para la función cerebral, el control de la circulación, la regulación osmótica y el metabolismo neuronal, así como lesión e inflamación de los astrocitos. Tales factores parecen estar implicados en la patogénesis de la manifestaciones neurológicas de la enfermedad (17-20).

En los pacientes con HE, el amonio se genera en el intestino, producto del catabolismo de las proteínas de la dieta, de la desaminación de la glutamina y por la acción de bacterias presentes en la flora intestinal (11, 21). La ureasa suministra a las bacterias el nitrógeno (N) necesario para la síntesis de aminoácidos. Este elemento se encuentra presente en el intestino como producto de la ingesta de una dieta rica en proteínas, por su parte, las bacterias intestinales producen amonio como producto de desecho, durante la digestión de proteínas. Consecuentemente, se produce un aumento en la concentración de amonio que, debido a la lesión de los hepatocitos que presentan los pacientes con HE y a un inadecuado funcionamiento del ciclo de la urea en el hígado, no puede ser totalmente metabolizado. En condiciones normales, el hígado metaboliza todo el amonio en urea, una molécula no tóxica que se elimina fácilmente en la orina.

\section{Metabolismo del amonio y origen de la fatiga central}

En condiciones normales, el cerebro metaboliza el amonio producido, pues los astrocitos en el cerebro son fuente abundante de la enzima glutamina sintasa 


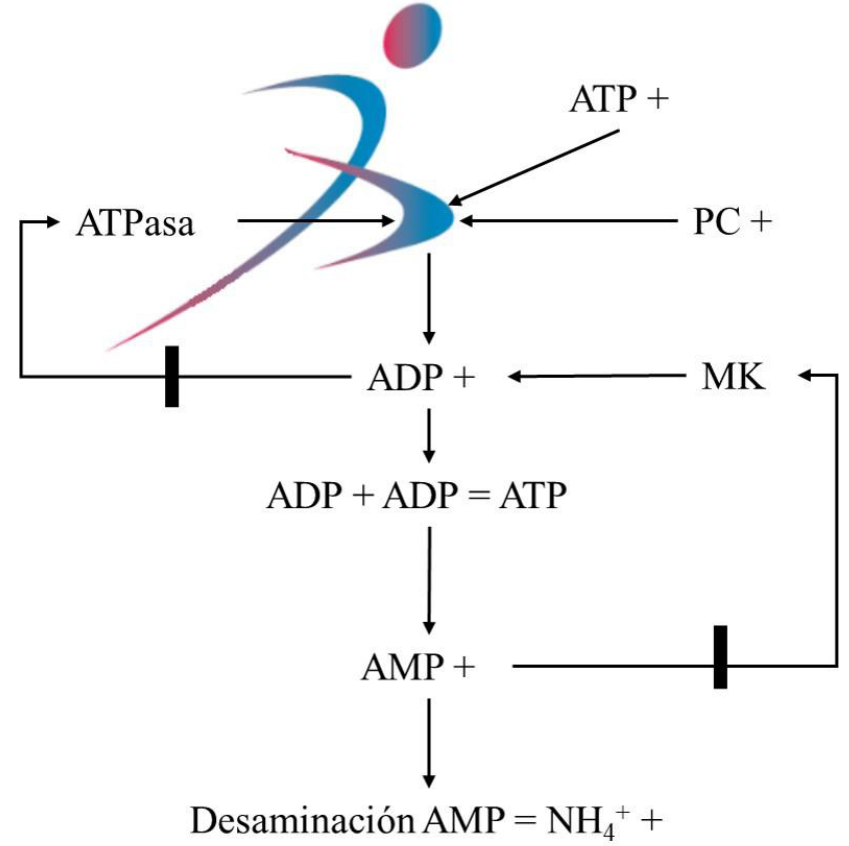

Figura 2. Producción de amonio en el músculo esquelético en ejercicio.

Durante el ejercicio de alta intensidad, se requiere de una cantidad energía (ATP y PC) para la contracción muscular. Como consecuencia de la reacción, se obtiene ADP, si este se acumula, bloquea la ATPasa. Del ADP acumulado se puede obtener ATP gracias a la acción de la enzima MK, como consecuencia de la reacción se obtiene AMP. Si este se acumula, bloquea la MK, por lo que la acumulación de AMP es inminente, cuya desaminación produce amonio.

*ATP: adenosin trifosfato, PC: fosfocreatina, ATPasa: enzima ATPasa, ADP: adenosin difosfato, MK: enzima mioquinasa, AMP: adenosin monofosfato, $\mathrm{NH}_{4}^{+}$: amonio, +: este símbolo significa cantidad, $\mid$ : este símbolo significa la reacción se inhibe o bloquea.

Fuente: Elaborado por autor

(GS), que convierte el amonio más glutamato en glutamina (glutamato $+\mathrm{NH}_{4}^{+} \rightarrow$ Glutamina), una molécula neutra no tóxica $(9,22)$. Posteriormente, la glutamina es llevada hasta el hígado, donde tiene lugar el proceso de desaminación del nitrógeno y, mediante el eficaz ciclo de la urea, el exceso de amonio es eliminado del organismo mediante la orina.

Sin embargo, debido a que el cerebro no tiene un eficaz ciclo de la urea, depende únicamente de la síntesis de glutamina para la eliminación del exceso de amonio (23). Por ello, en el cerebro se convierte gran parte del glutamato en glutamina. El glutamato es el principal neurotransmisor excitador del SNC, por su parte, la glutamina es precursor del ácido gammaaminobutírico (GABA) y, a su vez, el principal neurotransmisor inhibidor del SNC. Por lo tanto, no permite que las motoneuronas funcionen de forma adecuada y afecta el rendimiento motor, al originar fatiga central $(1,24)$. Asimismo, el amonio generado en el músculo esquelético, al ingresar al SNC, no permite que las motoneuronas funcionen de forma adecuada, afectan el rendimiento motor y causan fatiga central y periférica $(13,23)$. La fatiga central, en consecuencia, limita la capacidad del organismo para realizar actividades deportivas a velocidades o intensidades supra humanas.

\section{Ejercicio y sistema inmune adaptativo en el metabolismo del amonio}

El análisis del transporte de amonio ha permitido identificar, por una parte, una importante función del músculo esquelético en la eliminación de amonio (17) y, por otra, que el sistema inmune adaptativo está relacionado al metabolismo del amonio, mediante las células T. Estas son una fuente abundante de glutamato extracelular en el cerebro, que es tomado por los astrocitos y provoca la secreción de moléculas neuroprotectoras. Además, estas células protegen la eliminación de glutamato de los astrocitos, que se disminuye por el estrés oxidativo (25). Por tanto, la interacción entre las células $\mathrm{T}$ y los astrocitos juega un papel en el mecanismo molecular, pues ocasiona neuroprotección e impide la lesión en el SNC (25). Asimismo, el glutamato extracelular proveniente de las células T, combinado con $\mathrm{NH}_{4}^{+}$(producido por la contracción muscular), genera glutamina como producto de la reacción y favorece así la eliminación de glutamato de los astrocitos.

El recuento de células $\mathrm{T}$ o linfocitos $\mathrm{T}$ aumenta hasta $150 \%$ después de ejercicios de alta intensidad y corta duración. No obstante, este aumento parece estar más relacionado con personas sin entrenamiento que con atletas de alto nivel competitivo $(7,26)$. Durante ejercicios de resistencia de larga duración, como la maratón, las células $\mathrm{T}$ disminuyen transitoriamente; en cambio, en ejercicios de corta duración y moderada intensidad (alrededor de una hora o menos), no tiene efectos sobre las respuestas proliferativas de los linfocitos $T(7,26)$.

De alguna manera, la práctica del ejercicio físico regular induce adaptaciones metabólicas que hace 
que las altas concentraciones de amonio, perjudiciales para la salud, sean toleradas por los atletas. Como se mencionó, durante el ejercicio, estos deportistas presentan concentraciones de amonio en sangre superiores a los niveles considerados normales. La hiperamonemia está asociada, usualmente, con los síntomas de pacientes con HE; sin embargo, los atletas no presenten síntomas de dicha patología.

\section{Conclusiones}

Posiblemente, en el cerebro, el glutamato proveniente de las células $\mathrm{T}$ reacciona con el amonio producido por el musculo esquelético, durante ejercicios tanto de alta intensidad como de larga duración, generando glutamina como producto de la reacción, una molécula neutra no toxica. De esta manera, disminuye la fatiga central en atletas. No obstante, también podría deberse a la inducción de moléculas neuroprotectoras por parte de las células $\mathrm{T}$, que estarían generando neuroprotección e impidiendo la lesión de los astrocitos. Consecuentemente, la práctica del ejercicio físico regular induce adaptaciones metabólicas que juegan un papel importante en el metabolismo del amonio en el cerebro y, así, estaría generando neuroprotección contra las altas concentraciones de amonio, ya que los atletas durante el ejercicio con altas concentraciones de amonio no presentan los síntomas de pacientes con HE. La investigación científica referente a los efectos del amonio inducido por el ejercicio puede ofrecer una aplicación práctica en el tratamiento de la HE. No obstante, este tema requiere mayor explicación bioquímica y metabólica, así como estudios con adecuados diseños metodológicos, que permitan estudiar mejor el tema.

\section{Conflicto de intereses}

El autor declara no tener conflicto de interés, pues es independiente con respecto a las instituciones financiadoras y de apoyo. Además, manifiesta que durante la ejecución del trabajo y la redacción del manuscrito, no han incidido intereses o valores distintos a los propios de la investigación.

\section{Financiación}

Universidad Pedagógica y Tecnológica de Colombia, Facultad Seccional Chiquinquirá.

\section{Referencias}

1. Nybo L, Secher NH. Cerebral perturbations provoked by prolonged exercise. Prog
Neurobiol. 2004;72(4):223-261. doi:10.1016/j. pneurobio.2004.03.005

2. Lopéz J, Fernández A. Fisiología del ejercicio. Segunda edición. [Internet]. Madrid, España: Editorial Médica Panamericana; 2006 [citado 26 de marzo de 2018]. Recuperador a partir de: https://www.medicapanamericana.com/Libros/ Libro/3924/Fisiologia-del-Ejercicio.html

3. Entine J. Why black athletes dominate sports and why we're afraid to talk about it. [Internet]. New York Times: Public Affairs. 2000 [citado 26 de marzo de 2018]. Recuperado a partir de: https:// archive.nytimes.com/www.nytimes.com/books/ first/e/entine-taboo.html

4. Carvalho-Peixoto J, Alves RC, Cameron LC. Glutamine and carbohydrate supplements reduce ammonemia increase during endurance field exercise. Appl Physiol Nutr Me. 2007;32(6):11861190. doi: 10.1139/H07-091

5. Bessa A, Nissenbaum M, Monteiro A, Gandra PG, Nunes L, Bassini-Cameron A, et al. High-intensity ultraendurance promotes early release of muscle injury markers. Brit J Sport Med. 2008;42(11):889893. doi: 10.1136/bjsm.2007.043786

6. Bassini-Cameron A, Monteiro A, Gomes A, Werneck-de-Castro JP, Cameron L. Glutamine protects against increases in blood ammonia in football players in an exercise intensity-dependent way. Brit J Sport Med. 2008;42(4):260-266. doi: 10.1136/bjsm.2007.040378

7. Viru A, Viru M. Análisis y control del rendimiento deportivo [Internet]. Barcelona: Paidotribo; 2003 [citado 26 de marzo de 2018]. Recuperado a partir de: http://www.paidotribo.com/ficha.aspx?cod=00695

8. Hellsten Y, Richter EA, Kiens B, Bangsbo J. AMP deamination and purine exchange in human skeletal muscle during and after intense exercise. J Physiol. 1999;520:909-920. doi: 10.1111/j.14697793.1999.00909.x

9. McKee T, McKee JR. Bioquímica: las bases moleculares de la vida [Internet]. Estados Unidos: Mc Graw Hill Education; 2009 [citado 26 de marzo de 2018]. Recuperado a partir de: https://accessmedicina.mhmedical.com/Content. aspx?bookid $=1960 \&$ sectionid $=147707411$

10. Nelson DL, Cox MM. Lehninger Principles of Biochemistry [Internet]. Alemania: Grupo editorial W. H. Freeman; 2004 [citado 26 de marzo de 2018]. Recuperado a partir de: https://onlinelibrary.wiley. com/doi/abs/10.1002/cbf.1216 
11. Huizenga JR, Gips $\mathrm{CH}$, Tangerman A. The contribution of various organs to ammonia formation: a review of factors determining the arterial ammonia concentration. Ann Clin Biochem. 1996;33(1):2330. doi: 10.1177/000456329603300103

12. Casey A, Greenhaff PL. Does dietary creatine supplementation play a role in skeletal muscle metabolism and performance?. Am J Clin Nutr. [Internet]. 2000 [Acceso 26 mar 2018]; 72(2):607617. Disponible en: https://doi.org/10.1093/ ajcn/72.2.607S.

13. Bøyum A, Rønsen O, Tennfjord V, Tollefsen S, Haugen A, Opstad P, et al. Chemiluminescence response of granulocytes from elite athletes during recovery from one or two intense bouts of exercise. Eur J App Physiol. 2002;88(1-2):20-28. doi: 10.1007/s00421-002-0705-2

14. Wagenmakers AJ, Beckers EJ, Brouns F, Kuipers H, Soeters PB, Van Der Vusse GJ, et al. Carbohydrate supplementation, glycogen depletion, and amino acid metabolism during exercise. Am J PhysiolEndoc M. 1991;260(6):E883-890. doi: 10.1152/ ajpendo.1991.260.6.E883

15. Banister EW, Cameron BJC. Exercise-induced hyperammonemia: peripheral and central effects. Int J Sport Med. 1990;11(1):29-142. doi. org/10.1055/s-2007-1024864

16. Richter EA, Ruderman BN. AMPK and the biochemistry of exercise: implications for human health and disease. Biochem J. 2009;418(2):261275. doi: 10.1042/BJ20082055

17. Butterworth RF. Pathophysiology of hepatic encephalopathy: a new look at ammonia. Metab Brain Dis. 2002;17(4):221-227. doi: 10.1023/A:1021989230535

18. Felipo V, Butterworth RF. Neurobiology of ammonia. Neurobiol. 2002;67(4):259-279. doi: 10.1016/S0301-0082(02)00019-9
19. Ott P, Larsen FS. Blood-brain barrier permeability to ammonia in liver failure: a critical reappraisal. Neurochem Int. 2004;44(4):185-198. doi: 10.1016/ S0197-0186(03)00153-0

20. Córdoba J, Mínguez B. Hepatic encephalopathy. Semin Liver Dis. [Internet]. 2008 [Acceso 26 mar 2018]; 28(1):70-80. Disponible en: https://doi. org/10.1055/s-2008-1040322.

21. Romero-Gómez M. Role of phosphate-activated glutaminase in the pathogenesis of hepatic encephalopathy. Metab Brain Dis. [Internet]. 2005 [Acceso 26 mar 2018]; 20(4):319-325. Disponible en: https://doi.org/10.1007/s11011-005-7913-5.

22. BoronW,BoulpaepE. MedicalPhysiology[Internet]. USA: Elsevier Health Sciences; 2012 [citado 26 de marzo de 2018]. Recuperado a partir de: https:// www.elsevier.com/books/medical-physiology-2eupdated-edition/boron/978-1-4377-1753-2

23. Suarez I, Bodega G, Fernandez B. Glutamine synthetase in brain: effect of ammonia. Neurochem Int. 2002;41(2-3):123-142. doi: 10.1016/S01970186(02)00033-5

24. Nybo L, Dalsgaard MK, Steensberg A, Møller $\mathrm{K}$, Secher NH. Cerebral ammonia uptake and accumulation during prolonged exercise in humans. J Physiol. 2005; 563(1):285-290. doi: 10.1113/ jphysiol.2004.075838

25. Garg SK, Banerjee R, Kipnis J. Neuroprotective immunity: $\mathrm{T}$ cell-derived glutamate endows astrocytes with a neuroprotective phenotype. J Immunol. 2008;180(6): 3866-3873. doi: 10.4049/ jimmunol.180.6.3866

26. Córdova MA. Serie Blanca. Inmunidad y ejercicio físico. Fisiología del ejercicio. [Internet]. Madrid, España: Editorial Médica Panamericana; 2006 [citado 26 de marzo de 2018]. Recuperador a partir de: https:/www.medicapanamericana.com/Libros/ Libro/3924/Fisiologia-del-Ejercicio.html 